\title{
A New Technique to eliminate excessive copies of Cloud Data through Secure Key Hierarchy
}

\author{
Dr. Nileshkumar Gupta \\ A.K. Garg Engineering College \\ Ghaziabad
}

\begin{abstract}
Attribute-based Encryption has been ordinarily utilized in circulated computation wherever an information supplier re-appropriates his/her mixed data to a cloud professional association and may grant the information to customers having unequivocal capabilities (or properties). Regardless, the quality ABE structure does not backing secure elimination of excessive data, and that is vital for removal of duplicate copies of undefined knowledge, thus saving extra space and framework data transmission. During this paper, we tend to gift an attribute-based mostly limit system with secure elimination of excessive data during an ewer cloud setting, wherever a non-public cloud is in charge of duplicate acknowledgment associated an open cloud manages the limit. Differentiated and, therefore, the previous knowledge elimination of excessive data systems, our structure has two central focuses. Directly off the bat, it'd be wont to subtly bestow knowledge to customers by demonstrating access approaches instead of sharing unscrambling keys. Moreover, it brings home the portions of bacon the quality plan of linguistics security for knowledge protection. Whereas existing structures merely achieve it by representational process and additional delicate security thought. Besides, we tend to set forward a framework to vary a code text over one access approach into figure writings of the proportionate plaintext nonetheless below varied access game plans while not revealing the first plaintext.
\end{abstract}

Keywords: security, safe perusing, key confirmation, diminish deduplication.

\section{INTRODUCTION}

Distributed computing considerably encourages data suppliers World Health Organization have to be compelled to spread their data to the cloud while not revealing their subtle data to outsiders and may wish purchasers with precise authorizations to own the choice to urge to the data. This assumes data to be positioned away in jumbled way with getting to regulate methods to such associate scope that no-one apart from purchasers with properties (or certifications) of express assemblies will be able to decipher the encoded data. An associate coding strategy that come across this requirement is termed attribute-based coding, wherever a client's non-public key's associated with a property set, a letter is disorganized below associate appearance approach over tons of possessions, and a shopper will decipher a cipher text with its non-public key if its planning of traits satisfies the doorway strategy connected with this cipher text. Still, the quality ABE framework disregards to achieve secure deletion of excessive data, which may be a procedure to spare further space and system transfer speed by confiscating excess replacements of the disorganized data place away within the cloud. Then one more time, as way as we tend to may presumably grasp, existing developments for secure deduplication aren't supported property based mostly coding. Since Attribute-based Encryption and secure deletion of excessive data are generally applied on the distributed computing, it is tempting to structure a scattered storing background with the two possessions.

We take into account the concomitant scenario within structure of a character based mostly warehousing framework supporting securely deletion of excessive data of disorganized data within the cloud, within which, the darkness will not store any file over once although the element that it would get mixed reproductions of an identical record encoded below varied admittance methods. Associate data worker, Bob, means that to transfer a document $M$ to cloud and offer $M$ through purchasers having sure accreditations. Thus on do in and of itself, Bob encodes $\mathrm{M}$ below associate entrance strategy associate over tons of abilities and transfers the relating cipher text to the cloud, with the tip goal that lone purchasers whose provisions of properties satisfying the doorway strategy will decipher the cipher text. Subsequently, a different data supplier, Alice, handovers a cipher text for equivalent elementary document $\mathrm{M}$ nonetheless attributable to associate alternative admittance approach A0. Since the report is assigned during a disorganized structure, the cloud cannot understand that plain text regarding cipher text of Alice is adored that examination to Bob's, and also can hoard M double. Such a traced warehousing spends further space and correspondence transmission capability.

\section{RELATED WORK}

ABE (Attribute-Based Encryption):

Sahai \& Waters, given the thought of it and later on, Goyal et al. elaborate the key organization ABE (KP-ABE) \& cipher text strategy $\mathrm{ABE}(\mathrm{CP}-\mathrm{ABE})$ as the two complementary kinds of ABE.

Bettencourt, Sahai, \& Waters projected prime CP-ABE. Nevertheless, it is safe underneath the traditional assembly model. A CP$\mathrm{ABE}$ structure underneath additional established access arrangements is projected by Goyal et al. supported the amount of 
hypothetic suspicion. Thus, on beating the constraint that the attribute house scale is poly on the face of it restricted within the security boundary and therefore the attributes square measure mounted ahead, Rouselakis and Waters designed an outsized universe CP-ABE system underneath the prime-request gathering. During this research paper, the Rouselakis Waters framework is occupied because of hidden arrange for stable development.

Securely Deletion of excessive data: With the target of additional economic area for distributed storage administrations, Douceur et al. projected the principal declare adjusting privacy and proficiency in playacting deduplication referred to as united coding, wherever a message is encoded underneath a message-decided key with the objective that unclear plaintexts square measure disorganized to the equivalent cipher texts. For this case, if two clients transfer a similar document, the cloud employee will watch the comparable cipher texts and store only one duplicate. Executions and variations of synchronous coding were deployed. Thus on formalizing the accurate safety description for joined coding, Bellare gave a crypto logic crude named letter fastened coding and purpose by purpose several explanations to catch completely diverse safety requests. Abadi et al. excellence concluded the security classification by seeing the plain text conveyances relying upon the open boundaries of plans. This prototype was later reached out by Bellare by giving security to communications that square measure each connected and dependent on the open framework boundaries. Since message-bolted coding cannot avoid savaging power assaults wherever records falling into an accomplished set are recuperated, a style that provides secure deletion of excessive data reposition opposing beast power assaults was advanced Keelveedhi, Bellare and Ristenpart and acknowledged in a powerful framework referred to as server-helped coding for deletion of excessive data capability. During this research paper, a parallel strategy to it has been used to complete secure deletion of excessive data with relevancy the personal cloud within the stable development.

\section{PRELIMINARIES}

In following segment, we will audit important cryptographic thoughts and classifications that are to be utilized in later stage.

\subsection{Bilinear Couplings and Complexity Conventions}

Assume that Group on may be a probabilistic polynomial time calculation that inputs a security boundary $\lambda$, and yields a triplet $(\mathrm{P}, \mathrm{g}, \mathrm{p})$ wherever $\mathrm{P}$ maybe a gathering of request $\mathrm{g}$ that's created from $\mathrm{p}$, and $\mathrm{g}$ may be a prime. We tend to characterize ${ }^{\wedge} \mathrm{a}: \mathrm{P} \times \mathrm{P}$ $\rightarrow \mathrm{P} 1$ to be an additive guide the off probability that it's the attendant properties.

- Bilinear: for all $\mathrm{p} \in \mathrm{P}$, and $\mathrm{c}, \mathrm{d} \in \mathrm{Z} * \mathrm{~g}$, we've got ${ }^{\wedge} \mathrm{a}(\mathrm{pc}, \mathrm{gd})={ }^{\wedge} \mathrm{a}(\mathrm{p}, \mathrm{p}) \mathrm{cd}$.

- Non-degenerate: ^a(p,p) $6=1$.

We state that $\mathrm{P}$ may be an additive gathering if the gathering activity in $\mathrm{P}$ is profitably computable Associate in Nursing $\mathrm{d}$ there exists a gathering $\mathrm{P} 1$ and an effectively process able additive guide ${ }^{\wedge} \mathrm{a}: \mathrm{P} \times \mathrm{P} \rightarrow \mathrm{P} 1$ as higher than.

$$
\begin{array}{ll}
\text { given } \rightarrow-\mathrm{y}= \\
p, p^{\mu}, \\
p^{a^{i}}, p^{b^{i}}, p^{s . b_{j}}, p^{a^{i} b_{j}}, p^{a^{i} / b_{j}^{2}} & \forall(i, j) \in[f, f], \\
p^{a^{i} / b_{j}} & \forall(i, j) \in[2 f, f], i \neq f+1, \\
p^{a^{i} b_{j} / b_{j^{\prime}}^{2}} & \forall\left(i, j, j^{\prime}\right) \in[2 f, f, f], j \neq j^{\prime}, \\
p^{\mu a^{i} b_{j} / b_{j^{\prime}}}, p^{\mu a^{i} b_{j} / b_{j^{\prime}}^{2}} & \forall\left(i, j, j^{\prime}\right) \in[f, f, f], j \neq j^{\prime},
\end{array}
$$

It is onerous to acknowledge $\left(\rightarrow-\mathrm{y},{ }^{\wedge} \mathrm{a}(\mathrm{p}, \mathrm{p}) \mathrm{cf}+1 \mu\right)$ from $(\rightarrow-\mathrm{y}, \mathrm{Z})$, wherever $\mathrm{p} \in \mathrm{P}, \mathrm{Z} \in \mathrm{P} 1, \mathrm{c}, \mu, \mathrm{d} 1, \ldots, \mathrm{bf} \in \mathrm{Z} * \mathrm{~g}$ picked autonomously and systematically at impulsive.

\subsection{Symmetric Encryption}

Symmetric encryption schemes have keyspace KS and MS is made of the two algorithms: an encryption calculation SE. Ec(KS, $\mathrm{ms}$ ) produces a cipher text $\mathrm{C}$ on input a key KS $\in \mathrm{KS}$ and a message ms $\in \mathrm{MS}$, and a decoding calculation $\mathrm{SE}$. Dc(KS, C), which yields a communication ms or the disappointment image $\perp$ on input a key KS $\in \mathrm{KS}$ and any cipher text $\mathrm{C}$.

\subsection{Assurance Structure}

An assurance structure CME is made out of the accompanying 3 calculations boundary age calculation CPG which is taking a security boundary $\lambda$ as information and yields the open boundaries cpars, committal calculation Com which is taking the open boundaries cpars \& information $\mathrm{x}$ as information and yields a dedication com to $\mathrm{x}$ alongside a de-committal key dec, and a confirmation calculation that it acknowledges or 0 to demonstrate that it rejects. A responsibility plan ought to be both restricting, which implies that the decommit stage can effectively open to just one worth, and concealing, which implies that the submit stage doesn't uncover any data about $\mathrm{x}$. For $\mathrm{X} \in\{$ Hiding, Binding $\}$. 


\subsection{Access Arrangements \& Linear Secret Allocation Arrangements}

\section{Definition 1: (Access Arrangements)}

Let $\{A 1, \ldots, A n\}$ be a set of parties. An assortment $B \subseteq 2\{A 1, \ldots, A n\}$ is monotone in the event that $\forall C, D$ : in the event that $C \in A n$ and $\mathrm{C} \subseteq \mathrm{D}$, at that point $\mathrm{D} \subseteq \mathrm{B}$. $\mathrm{B}$ (monotone) get to structure is a (monotone) assortment An of non-void subsets of $\{\mathrm{A} 1, \ldots, \mathrm{An}\}$, i.e., $\mathrm{B} \subseteq 2\{\mathrm{~A} 1, \ldots, \mathrm{An}\} \backslash\{\emptyset\}$. The sets are known as the approved sets, and the sets not in An are known as the unapproved sets.

\section{Definition 2: (Linear Secret Allocation Arrangements)}

Leave A alone a lot of gatherings. Let $\mathrm{G}$ be a grid of size $1 \times \mathrm{n}$. Let $\rho:\{1, \ldots, 1\} \rightarrow \mathrm{R}$ be a capacity that plots a line to a group for naming. Leave $\mathrm{r}$ alone any prime digit. A mystery distribution plan $\Pi$ terminated a lot of gatherings $\mathrm{R}$ is a direct mystery sharing plan (LSSS) over Zr if

1) The offers for each gathering structure a vector over $\mathrm{Zr}$.

2) There is a grid $G$ with one-lines and $n$ sections called the offer creating lattice for $\Pi$.

\section{SYSTEM ARCHITECTURE AND SECURITY MODEL}

In this area, we portray the framework design and the proper meaning of ciphertext-strategy trait based capacity framework supporting secure deduplication.

\subsection{System Architecture}

The engineering of our quality primarily based reposition framework with secure deduplication has appeared in Figure during which four components area unit included: data suppliers, appropriate authority (CA), cloud, and purchasers. An associate degree data provider has to re-appropriate her/his data to the cloud and supply it with purchasers having sure accreditations. The CA provides every consumer associate degree unscrambling key connected with its arrangement of qualities. The cloud contains associate degree open cloud that is answerable for data reposition and a personal cloud that plays out a sure calculation, as an example, tag checking. Once causing a record to reposition demand, each data provider at the start marks label $\mathrm{T}$ and mark L connected with statistics, and associate degreed later struggles the data below an entry arrangement over heaps of characteristics. Besides, all data supplier creates a symptom pf on the connection of the label T, the mark L and also the encoded message ct3

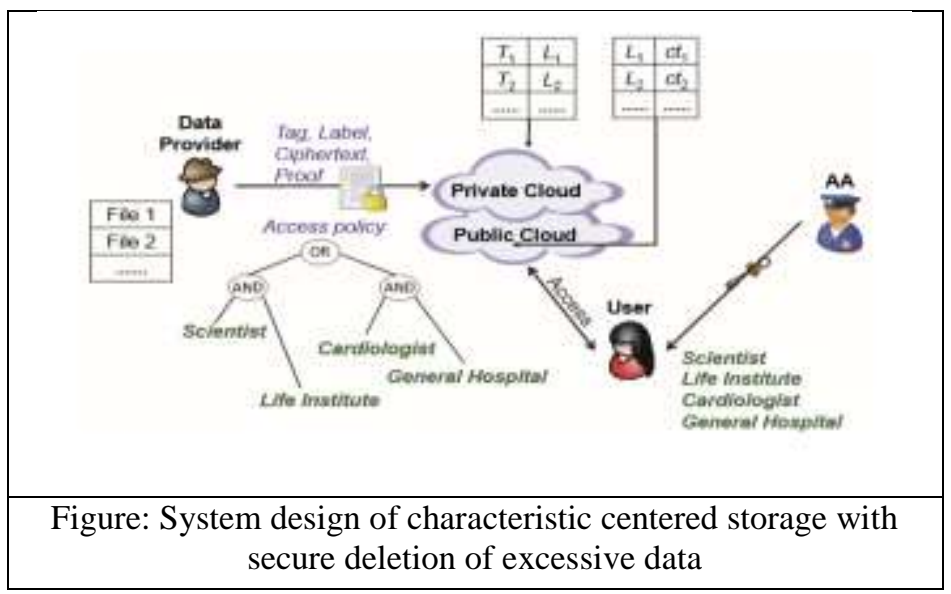

\section{ATTRIBUTE-BASED STORAGE WITH SECURE DEDUPLICATION}

In this segment, we depict a stable development of a trait-based capacity framework supporting secure deduplication, examine its security, and show its exhibition from theoretical and trial investigation.

\subsection{Construction}

- Decrypt. This calculation takes the open boundary standards, a ciphertext $(\mathrm{M}, \rho), \mathrm{E}, \mathrm{B}, \mathrm{C},\{\mathrm{Ci}, \mathrm{Di}, \mathrm{Ei}\} \mathrm{i} \in[1,1]$ with the relating name $\mathrm{K}$ and a private key sA for a property set An as the info. Assume that a particular set A fulfills the entrance structure (M, $\rho$ ). Characterize $I$ as $I=\{i: \rho(i) \in A\}$. Indicate by $\{$ wi $\in Z p\} i \in I$ a lot of constants with the end goal that if $\{$ vi $\}$ are legitimate portions of any mystery $\mu$ as per $(\mathrm{M}, \rho)$, then PieI wivi $=\mu$. It figures the message $\mathrm{M}$ as ${ }^{\wedge} \mathrm{a}(\mathrm{B}, \mathrm{s} 01) \mathrm{Qi} \in \mathrm{I}\left({ }^{\wedge} \mathrm{a}(\mathrm{Ci}, \mathrm{s} 02)^{\wedge} \mathrm{a}(\mathrm{Di}, \mathrm{s}(\mathrm{i}) 1)^{\wedge} \mathrm{e}(\mathrm{Ei}, \mathrm{s}(\mathrm{i})\right.$

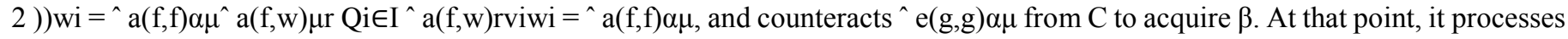
$\mathrm{M}=\mathrm{SE} . \operatorname{Dc}(\mathrm{G}(\beta), \mathrm{E})$. On the off chance that $\mathrm{fg} 1(\mathrm{M}) \mathrm{hg} 0(\beta)=\mathrm{L}$, it yields M. Else, it yields a disappointment symbol $\perp$.

\subsection{Security}

Next, we demonstrate that the proposed stockpiling framework safeguards the security of the encoded information as far as open Cloud and private Cloud, separately.

\section{Theorem}

Expecting that $(\mathrm{q}-1)$ supposition clamps in F, SE is a protected single key encryption plan, and L is created succeeding a safe duty conspire, at that point, the proposed characteristic based hoarding background with secure deletion of excessing data is specifically 
unclear in regards to the perspective on the open Cloud. Confirmation. The Rouselakis Waters plot is identified to be pointedly ambiguous accepting that $(q-1)$ presumption grips in F. Our verification for Theorem 1 generally surveys that in excluding that in test stage, E* and $\mathrm{L} *=\mathrm{fg} 1(\mathrm{M} * \mathrm{~b}) \mathrm{hg} 0(\beta)$ will be added to the first test ciphertext. Note that $\mathrm{E} *$ won't unveil any data about $\mathrm{M} * \mathrm{~b}$ because of safety of the fundamental SE scheme, L* won't enlighten any data concerning M*b because of the safety of the basic responsibility plot.

\subsection{Implementation}

My project implements using with modules.
i) Data Provider
ii) Cloud
iii) Deletion of
iv) Attribute Authority

\subsubsection{Data Provider}

Information supplier transferring document to cloud with tag, name and security key, the proposed plot ensures information uprightness against any label irregularity assault. Accordingly, security is improved in the proposed plot.

\subsubsection{Cloud}

Secure Deduplication to spare storing universe for distributed storing administrations, Douceur et al. The main answer for adjusting classification and productivity in deletion of excessive data called joined encryption, where a communication is scrambled under a message contingent key, so indistinguishable plain texts are encoded to the equivalent ciphertexts. For this situation, if two clients transfer a similar record, the cloud worker can perceive the equivalent ciphertexts and store just one duplicate of them. Which may disregard the protection of the information if the cloud worker can't be completely trusted. This is a customer who possesses information and wishes to transfer it into the distributed storage to spare expenses. An information proprietor scrambles the data and re-appropriates it to the distributed storage with its file data, that is, a tag.

\subsubsection{Deduplication}

Information deduplication is a particular information pressure method for dispensing with copy duplicates of rehashing information. Related and to some degree, equal terms are canny (information) pressure and single-occurrence (information) stockpiling. This procedure is utilized to improve capacity use and can likewise be applied to organize information moves to lessen the number of bytes that must be sent. In the deduplication procedure, one of a kind pieces of information, or byte designs, are distinguished and put away during a process of examination. Deduplication strategies exploit information similitude to distinguish similar information and decrease the extra room. Conversely, encryption calculations randomize the encoded documents to make ciphertext undefined from hypothetically irregular information.

\subsubsection{Attribute Authority}

The AA gives each client an unscrambling key associated with client set of qualities At the client-side, every client can download a thing, and decode the ciphertext with the property based private key created by the AA if this present client's characteristic set fulfills the entrance structure.

\section{EXPECTED RESULTS DISCUSSION}

In this segment, we give further elaboration on the two principle methods we presented in this paper.

\subsection{Adjustable Characteristic Centered Encryption}

Lai et al. introduced crypto logical crude referred to as multipurpose CP-ABE, wherever a semi-believed intercessor is carried into the locale of CP-ABE. The intercessor, given a background full hidden entry key, will modify any code text beneath one admittance approach into cipher texts of the equivalent plain text beneath another access method while not learning any knowledge regarding the plaintext throughout the modification procedure. However, this strategy for utilizing a single secret entry key for all cipher texts is remarkably insecure, since if the only key's undermined.

The safety for the background is going to be thoroughly damaged. An associate ill-disposed consumer utilizing the undermined secret entrance key will recover a cipher text into associate entry arrangement that his/her characteristics fulfill. During this manner, it will get the plain text not planned for him/her. Moreover, the hidden entrance key is formed by the AA UN agency as of currently controls the decipherment keys within the framework. Therefore it's enticing to decrease its capability in dominant cryptography. Not the least bit like that in our procedure is coordinated with the tip goal that every hidden entrance key should be used to alter its relating ciphertext. On these lines, even sooner or later, a secret entrance key's contained, the hurt is strained to 1 message. At a significant level, our strategy carries an associate with another approach to fabricate versatile $\mathrm{CP}$-ABE frameworks from an alternate perspective.

\subsection{Deduplication in Hybrid Cloud}

Take care of this issue, a more vulnerable safety idea called defense under picked dissemination assaults [8] was advanced under the supposition that the information message is adequately erratic. Not quite the same as the current technique for characterizing a more vulnerable security idea for the distributed storage framework with secure deduplication, half and half cloud design, comprising of a couple of open and private mists, is presented in our capacity framework to such an extent that the semantic security gets attainable for the open cloud. This structure of twin mists has been generally received practically speaking, where the security 
of the open cloud for the most part goes up against a bigger number of difficulties than that of the private cloud, and consequently it is alluring to have more grounded information secrecy insurance at the open cloud side. We accept that the cross breed cloud engineering is a promising way to deal with capacity frameworks with deletion of excessive data, in which the scrambled information is re-appropriated to the open cloud while deletion of excessive data checking is taken care of by the private cloud.

\section{CONCLUSION}

Attribute-Based Encryption is being extensively useful in disseminated processing where data suppliers redistribute their mixed data to cloud and they can confer the data to customers having shown qualifications. Then again, deletion of excessive data is a huge technique to save the additional area and framework move speed, which discards duplicate copies of indistinct data. In any case, the standard ABE structures don't provision safe deletion of excessive data, which marks them overpriced to be connected in some business amassing organizations. In this research paper, we showed a novel method to manage comprehend a quality based limit system supporting safe deletion of excessive data. Our capability arrangement has operated under a creamer cloud building, in which a private cloud controls the sum and open cloud manages limit. Resulting to getting a limit request, the private cloud first draughts the legitimacy of the moved thing with the associated proof. If the proof is real, the private cloud turns a mark establishing approximation to see if comparative data covered up cipher text has been taken care of. Accepting this is the situation, at whatever point it is significant, it recuperates cipher text to a cipher text of comparable plain text over a passageway approach which is the affiliation set of both access systems. The proposed amassing system acknowledges two imperative focal points. Directly off the bat, it might be used to subtly give data to various customers by deciding a passageway approach as conflicting to sharing the unscrambling key. Moreover, it achieves the standard thought of semantic safety while obtainable deletion of excessive data contrives simply achieve it under a more delicate safety thought.

\subsection{Results}

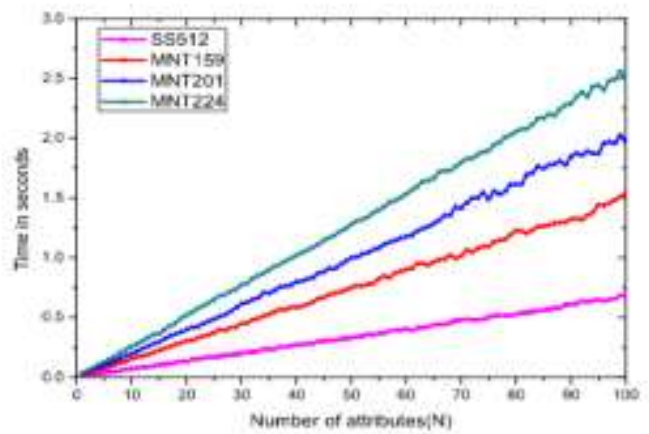

(a) KeyGen

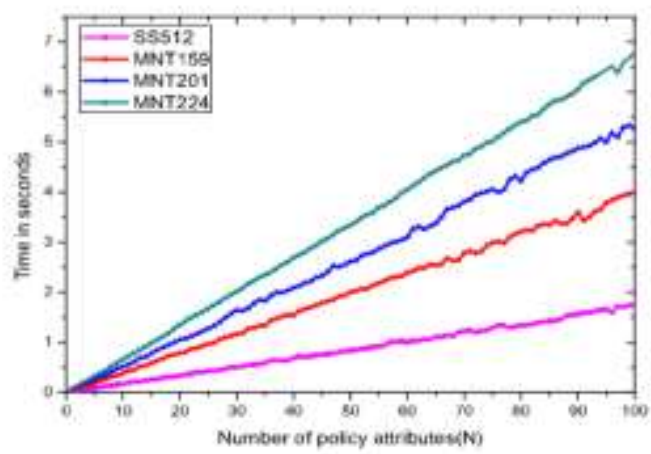

(c) Re-encrypt

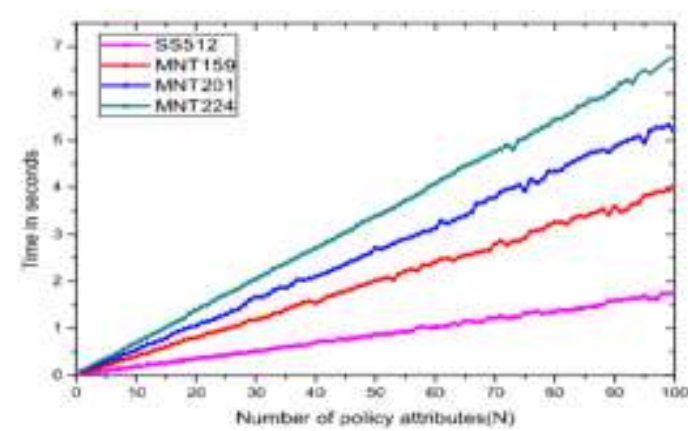

(b) Encrypt

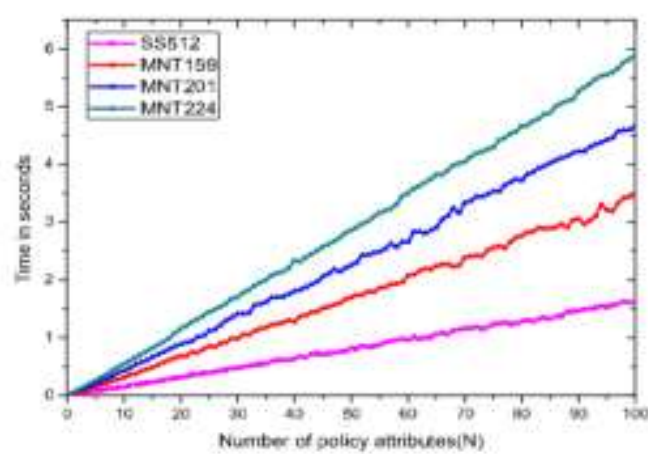

(d) Decrypt

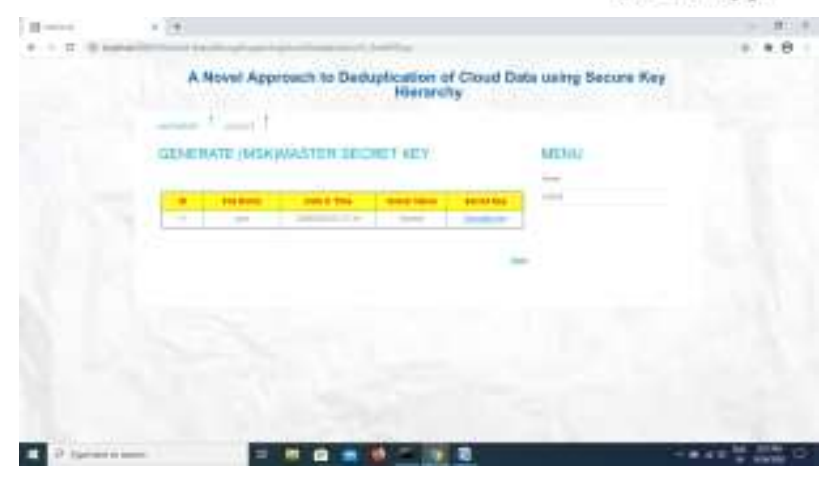




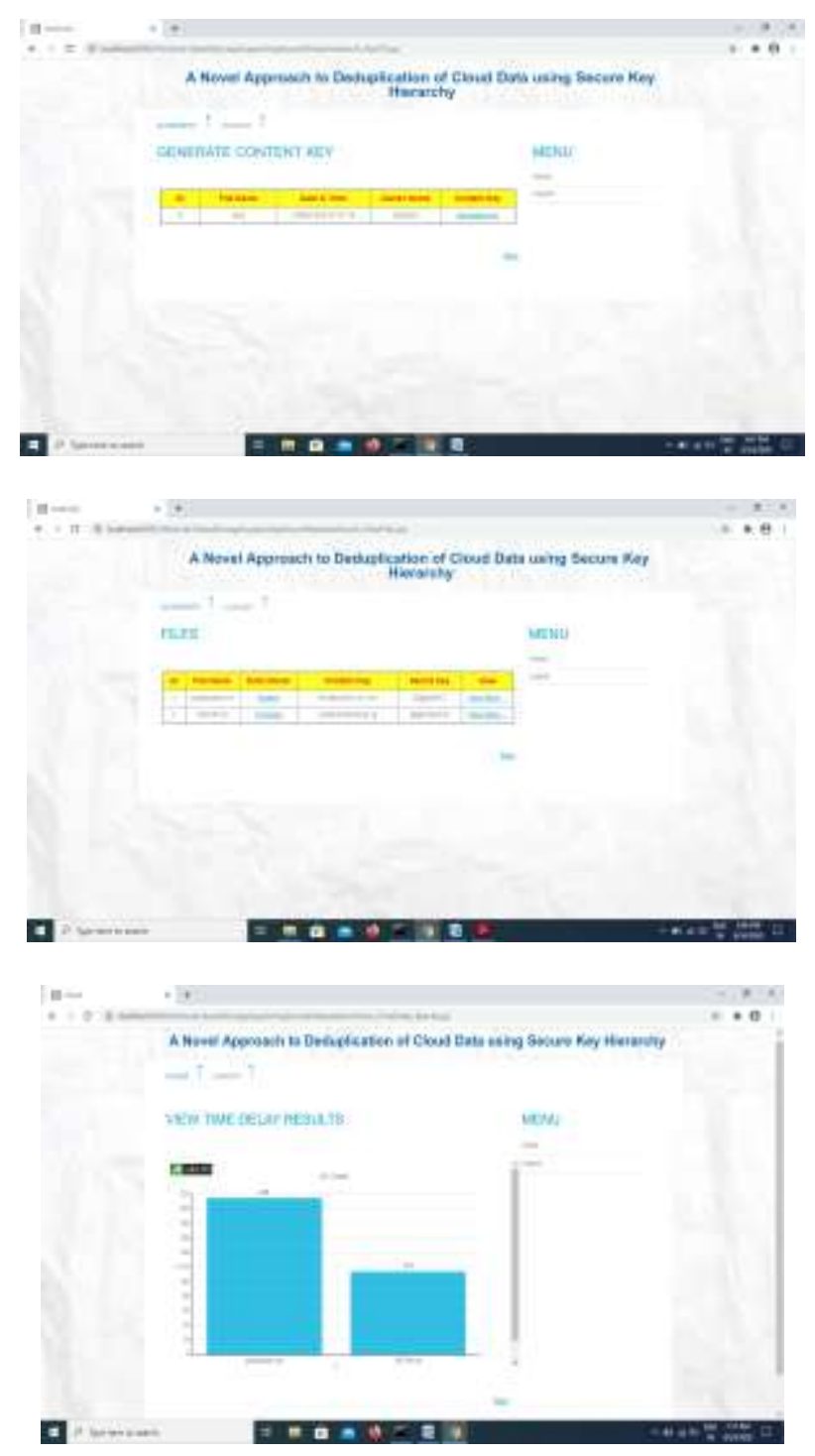

\section{REFERENCES}

[1] D. Quick and K. R. Choo, "Google drive: Forensic analysis of data remnants," J. Network and Computer Applications, vol. 40, pp. 179- 193, 2014.

[2] Hendrik Graupner, Kennedy A Torkura, Muhammad Sukmana, Christoph Meinel, "Secure Deduplication on Public Cloud Storage", ICBDC 2019: Proceedings of the 2019 4th International Conference on Big Data and Computing, May 2019 Pages 34-41, https://doi.org/10.1145/3335484.3335502"

[3] Liu Zhenhua, Kang Yaqian, Li Chen, Fan Yaqing, Hybrid cloud approach for block-level deduplication and searchable encryption in large universe, The Journal of China Universities of Posts and Telecommunications, Volume 24, Issue 5, 2017, Pages 23-34, ISSN 1005-8885, https://doi.org/10.1016/S1005-8885(17)60230-9

[4] S. Annie Joice, M. A. Maluk Mohamed, Cloud Storage: A Review on Secure Deduplication and Issues, Journal of Internet Technology Volume 20 (2019) No.3, DOI: 10.3966/160792642019052003019

[5] Sara Abdel Razek, Dr.Heba El-Fiqi, Prof. Dr. Ibrahim Mahmoud "Cloud Storage Forensics: Survey", International Journal of Engineering Trends and Technology (IJETT), V52(1),22-35 October 2017. ISSN:2231-5381. www.ijettjournal.org. published by seventh sense research group

[6] M. Bellare, S. Keelveedhi, and T. Ristenpart, "Message-locked encryption and secure deduplication," in Advances in Cryptology - EUROCRYPT 2013, 32nd Annual International Conference on the Theory and Applications of Cryptographic Techniques, Athens, Greece, May 26-30, 2013. Proceedings, ser. Lecture Notes in Computer Science, vol. 7881. Springer, 2013, pp. 296-312.

[7] K. R. Choo, M. Herman, M. Iorga, and B. Martini, "Cloud forensics: State-of-the-art and future directions," Digital Investigation, vol. 18, pp. 77-78, 2016.

[8] Chinmay Patil, Shubham Kasabe, Ronik Mahajan, Ajay Indani, Prof. V.V. Waykule, "Attribute based Storage to avoid duplicate files on cloud", International Journal of Advance Research in Engineering, Science \& Technology, Volume 5, Issue 4, April-2018

[9] 1. M. Jiang, Y. Hu, H. Lei, B. Wang, Q. Lai, "Lattice-based certificateless encryption scheme", Frontiers of Computer Science, Vol. 8, p. 828, 2014, DOI: https://doi.org/10.1007/s11704-014-3187-6 